Pacific Journal of Mathematics

STOCHASTIC INTEGRATION IN FOCK SPACE 


\title{
STOCHASTIC INTEGRATION IN FOCK SPACE
}

\author{
V. S. SUNDER
}

In this paper, using purely Hilbert space-theoretic methods, an analogue of the Itô integral is constructed in the symmetric Fock space of a direct integral $\mathfrak{S}$ of Hilbert spaces over the real line. The classical Itô integral is the special case when $\mathscr{S}=L^{2}[0, \infty)$. An explicit formula is obtained for the projection onto the space of 'non-anticipating functionals', which is then used to prove that simple non-anticipating functionals are dense in the space of all non-anticipating functionals. After defining the analogue of the Itô integral, its isometric nature is established. Finally, the range of this 'integral' is identified; this last result is essentially the Kunita-Watanabe theorem on square-integrable martingales.

Preliminaries. (a) Symmetric Fock space: If $\mathfrak{S}$ is a (complex) Hilbert space, the symbol $\mathfrak{S}^{(s) n}$ will denote the Hilbert space of symmetric tensors of rank $n$; alternatively, $\mathscr{S}^{(s) n}$ is the closed subspace of $\otimes^{n} \mathfrak{E}$ spanned by $\{x \otimes \cdots \otimes x: x \in \mathfrak{S}\}$. (In the sequel, the $\operatorname{symbol} \operatorname{sp} S$ will denote the closed subspace spanned by the set $S$ of vectors.) By convention, $\mathfrak{S}^{(s) 0}=\mathbf{C}$. We shall also write $\otimes^{n} x$ for $x \otimes \cdots \otimes x$, with the convention that $\otimes{ }^{0} x=1$.

The symmetric Fock space over $\mathfrak{g}$, is by definition, the Hilbert direct sum

$$
\Gamma(\mathfrak{S})=\bigoplus_{n=0}^{\infty} \mathfrak{S}^{(s) n}
$$

If $x \in \mathfrak{S}$, then $\Gamma(x)$ will denote the 'exponential' vector in $\Gamma(\mathfrak{S})$ defined by

$$
\Gamma(x)=\left(1, x, \frac{\otimes^{2} x}{\sqrt{2 !}}, \ldots, \frac{\otimes^{n} x}{\sqrt{n !}}, \ldots\right) .
$$

The following are easily verified:

$$
\Gamma(\mathfrak{S})=\operatorname{sp}\{\Gamma(x): x \in \mathfrak{S}\}
$$

and

$$
\langle\Gamma(x), \Gamma(y)\rangle=\exp \langle x, y\rangle, \quad x, y \in \mathfrak{S} .
$$


The symbol $\Omega$ is reserved for the 'vacuum' vector: $\Omega=\Gamma(0)=$ $(1,0,0, \ldots)$.

If $\mathfrak{S}_{1}$ and $\mathfrak{S}_{2}$ are Hilbert spaces, it follows from (1) that the correspondence

$$
\Gamma\left(\left(x_{1}, x_{2}\right)\right) \leftrightarrow \Gamma\left(x_{1}\right) \otimes \Gamma\left(x_{2}\right)
$$

extends to a canonical unitary isomorphism of Hilbert spaces:

$$
\Gamma\left(\mathfrak{S}_{1} \oplus \mathfrak{S}_{2}\right) \cong \Gamma\left(\mathfrak{S}_{1}\right) \otimes \Gamma\left(\mathscr{S}_{2}\right) \text {. }
$$

If $A$ is a contraction on $\mathfrak{S}$ (i.e., $A$ is an operator with $\|A\| \leq 1$ ), there exists a unique contraction $\Gamma(A)$ on $\Gamma(\mathfrak{S})$ such that $\Gamma(A) \Gamma(x)=\Gamma(A x)$ for all $x$ in $\mathfrak{S}$. (In fact, $\Gamma(A)=\oplus_{n=0}^{\infty}\left(\otimes^{n} A\right)$ ). If $A$ and $B$ are contractions on $\mathfrak{E}$, it is clear that

$$
\Gamma(A B)=\Gamma(A) \Gamma(B) ; \quad \Gamma(A)^{*}=\Gamma\left(A^{*}\right) .
$$

In particular, if $A$ is a projection, so also is $\Gamma(A)$.

(b) Continuous tensor products: If $(X, \mathscr{B}, \mu)$ is a measure space and $\mathfrak{S}=\int_{X}^{\oplus} \mathfrak{S}(t) \mu(d t)$ is a direct integral of Hilbert spaces over $X$ (cf. [2] for definition and basic facts about direct integrals), then, for each $M$ in $\mathscr{B}$, the operator of multiplication by $\chi_{M}$ will be denoted by $P(M)$. Thus, $M \rightarrow P(M)$ is the canonical spectral measure in $\mathfrak{S}$. If $\mathscr{H}=\Gamma(\mathfrak{S})$, we shall use the symbol $E(M)$ for $\Gamma(P(M))$. By the last remark in (a), each $E(M)$ is a projection; further, (2) implies that if $M \subseteq N$, then $E(M) \leq$ $E(N)$. Further, we shall write $\mathfrak{S}(M)=P(M) \mathfrak{S}$ and $\mathscr{H}(M)=E(M) \mathscr{H}$. Then, $\mathscr{H}(M)$ can be naturally identified with $\Gamma(\mathfrak{S}(M))$, and it is easy to see that $\mathscr{H}(M)=\left\{\left(f_{n}\right)_{n=0}^{\infty} \in \mathscr{H}: f_{n} \in \mathfrak{S}(M)^{(s) n}\right.$ for all $\left.n\right\}$.

If $M$ and $N$ are disjoint sets in $X$, then $\mathscr{S}(M \cup N) \cong \mathscr{S}(M) \oplus \mathscr{S}(N)$, and so, there exists a canonical unitary operator (cf. (a))

$$
U_{M, N}: \mathscr{H}(M) \otimes \mathscr{H}(N) \rightarrow \mathscr{H}(M \cup N) .
$$

(If $x \in \mathfrak{S}_{\mathfrak{g}}(M), y \in \mathfrak{S}_{\mathcal{E}}(N), U_{M, N}(\Gamma(x) \otimes \Gamma(y))=\Gamma(x+y)$.) The following properties of the $U_{M, N}$ 's are easily established (by verifying them on exponential vectors).

Proposition ( $U$ ). (i) If $L, M$ and $N$ are disjoint Borel sets in $X$, the following diagram of Hilbert spaces and unitary operators is commutative:

$$
\begin{array}{ccc}
\mathscr{H}(L) \otimes \mathscr{H}(M) \otimes \mathscr{H}(N) & \stackrel{1_{\mathscr{H}(L)} \otimes U_{M, N}}{\rightarrow} & \mathscr{H}(L) \otimes \mathscr{H}(M \cup N) \\
\downarrow U_{L, M} \otimes 1_{\mathscr{H}(N)} & & \downarrow U_{L, M \cup N} \\
\mathscr{H}(L \cup M) \otimes \mathscr{H}(N) & \stackrel{U_{L \cup M, N}}{\rightarrow} & \mathscr{H}(L \cup M \cup N)
\end{array}
$$


(ii) If $M \subseteq N$, then $\mathscr{H}(M) \subseteq \mathscr{H}(N)$ and

$$
U_{M, N \backslash M}(f \otimes \Omega)=f, \quad f \in \mathscr{H}(M) .
$$

(Note that $\Omega \in \mathscr{H}(L)$ for all $L \in \mathscr{B}$.)

Briefly, $\mathscr{H}$ has a continuous tensor product structure over $X$ (cf. [1] and [6]).

In case $X=[0, \infty), \mu$ is Lebesgue measure and $\mathscr{\mathcal { S }}=L^{2}[0, \infty)$, it is known that $\mathscr{H}=\Gamma(\mathscr{S})$ can be identified with $L^{2}(\mathscr{C}, P)$, where $\mathscr{C}=\{f \in$ $C[0, \infty): f(0)=0\}$ and $P$ is the Wiener (probability) measure defined on the $\sigma$-algebra generated by point-evaluations. Explicitly, the correspondence is given by

$$
\Gamma(\phi) \leftrightarrow \exp \left(\int \phi(t) d w(t)-\frac{1}{2} \int \phi(t)^{2} d t\right),
$$

where $\phi \in L^{2}[0, \infty)$ and the first integral on the right is the Wiener integral (cf. [6]).

The text. In the sequel, the notation and terminology will be exactly as in (b) above. We shall further restrict ourselves to the case where

(a) $X=\mathbf{R}$

(b) $\mathscr{B}$ is the $\sigma$-algebra of Borel sets in $\mathbf{R}$; and

(c) $\mu$ is a non-atomic, positive, $\sigma$-finite measure defined on $\mathscr{B}$. Thus,

$$
\mathscr{S}=\int_{\mathbf{R}}^{\oplus} \mathfrak{S}(t) \mu(d t) ; \quad \mathscr{H}=\Gamma(\mathfrak{S})
$$

For any $t$ in $\mathbf{R}$, we shall use the abbreviations $P_{t}, E_{t}, \mathfrak{S}_{t}$ and $\mathscr{H}_{t}$ respectively for $P(-\infty, t], E(-\infty, t], P_{t} \mathfrak{S}$ and $E_{t} \mathscr{H}$. The non-atomicity of $\mu$ ensures that inclusion or exclusion of one or both end-points of intervals is irrelevant. (Thus, $P_{t}=P(-\infty, t)$.) Further, the non-atomicity of $\mu$ implies that $\left\{P_{t}\right\}$ and $\left\{E_{t}\right\}$ are strongly continuous one-parameter families of projections.

The symbol $W$ will be reserved for the natural (isometric) inclusion of $\mathfrak{S}$ in $\mathscr{H}$ :

$$
W x=(0, x, 0,0, \ldots) .
$$

The map $W$ clearly satisfies

$$
\begin{aligned}
& W(\mathfrak{S}(M)) \subseteq \mathscr{H}(M), \quad M \in \mathscr{B}, \quad \text { and } \\
& \langle W x, \Omega\rangle=0, \quad x \in \mathscr{S} .
\end{aligned}
$$


In case $\mathfrak{S}=L^{2}[0, \infty)$ and $\mathscr{H}=L^{2}(\mathscr{C}, P)$, it can be verified that $W$ is just the Wiener integral: $W \phi=\int \phi(t) d w(t)$. In order to define the analogue of the Itô integral, we begin with the following:

Definition 1. A non-anticipating tensor (abbreviated to n.a.t. in the sequel) is an element of the closed subspace $\mathfrak{N}$ of $\mathscr{H} \otimes \mathfrak{F}$ defined by

$$
\mathfrak{N}=\left\{\phi \in \mathscr{H} \otimes \mathfrak{H}_{\mathscr{B}}:\left(1_{\mathscr{H}} \otimes P_{t}\right) \phi=\left(E_{t} \otimes P_{t}\right) \phi \forall t\right\}
$$

EXAmple 2. Let $a \in \mathbf{R}, f \in \mathscr{H}(-\infty, a], x \in \mathfrak{S}_{\mathfrak{g}}(a, \infty)$, and let $\phi=f \otimes$ $x$. Then $\phi$ is a n.a.t., since $\left(1_{\mathscr{H}} \otimes P_{t}\right) \phi=\left(E_{t} \otimes P_{t}\right) \phi=0$ if $t \leq a$, while if $t>a$,

$$
\left(E_{t} \otimes P_{t}\right) \phi=E_{t} f \otimes P_{t} x=f \otimes P_{t} x=\left(1_{\mathscr{H}} \otimes P_{t}\right) \phi .
$$

Definition 3. A n.a.t. of the sort described in Example 2 will be called an elementary n.a.t.; a finite linear combination of elementary n.a.t.s will be called a simple n.a.t.

The following elementary result is recorded here for later use.

Proposition 4. If $\phi \in \mathfrak{N}$ and $-\infty<a \leq b<\infty$, then

$$
\left(1_{\mathscr{H}} \otimes P(a, b]\right) \phi=\left(E_{b} \otimes P(a, b]\right) \phi .
$$

Proof.

$$
\begin{aligned}
\left(1_{\mathscr{H}} \otimes P(a, b]\right) \phi & =\left(1_{\mathscr{H}} \otimes P_{b}-1_{\mathscr{H}} \otimes P_{a}\right) \phi \\
& =\left(E_{b} \otimes P_{b}-E_{a} \otimes P_{a}\right) \phi, \quad \text { since } \phi \in \Re .
\end{aligned}
$$

Hence

$$
\begin{aligned}
\left(E_{b} \otimes P(a, b]\right) \phi & =\left(E_{b} \otimes 1_{\mathfrak{Q}}\right)\left(E_{b} \otimes P_{b}-E_{a} \otimes P_{a}\right) \phi \\
& =\left(E_{b} \otimes P_{b}-E_{b} E_{a} \otimes P_{a}\right) \phi \\
& =\left(E_{b} \otimes P_{b}-E_{a} \otimes P_{a}\right) \phi=\left(1_{\mathscr{H}} \otimes P(a, b]\right) \phi
\end{aligned}
$$

by the previous equality, and the proof is complete.

We now wish to obtain a formula for the projection of $\mathscr{H} \otimes \mathfrak{S}$ onto $\mathfrak{N}$, which will henceforth be denoted by $Q$. However, some notation should be established first.

Let $J=\left\{\left(t_{0}, t_{2}, \ldots, t_{n}\right):-\infty<t_{0}<t_{1}<\cdots<t_{n}<\infty, \quad n=\right.$ $1,2, \ldots\}$.The set $J$ is a directed set with respect to the partial order defined by

$$
\left(t_{0}, \ldots, t_{n}\right) \leq\left(s_{0}, \ldots, s_{n}\right) \quad \text { iff } \quad\left\{t_{0}, \ldots, t_{n}\right\} \subseteq\left\{s_{0}, \ldots, s_{m}\right\} .
$$


If $\Delta=\left(t_{0}, \ldots, t_{n}\right) \in J$, define

$$
Q_{\Delta}=\sum_{i=1}^{n} E\left(-\infty, t_{i-1}\right] \otimes P\left(t_{i-1}, t_{i}\right]
$$

Since the projections $\left\{P\left(t_{i-1}, t_{i}\right]: i=1, \ldots, n\right\}$ are mutually orthogonal it follows that $Q_{\Delta}$, being a sum of mutually orthogonal projections, is itself a projection with

$$
\operatorname{ran} Q_{\Delta}=\bigoplus_{i=1}^{n} \mathscr{H}\left(-\infty, t_{i-1}\right] \otimes \mathfrak{S}\left(t_{i-1}, t_{i}\right] .
$$

LEMMA 5. $\left\{Q_{\Delta}: \Delta \in J\right\}$ is a monotone net of projections; i.e., if $\Delta$, $\Delta^{\prime} \in J$ and $\Delta \leq \Delta^{\prime}$, then $Q_{\Delta} \leq Q_{\Delta^{\prime}}$.

Proof. It clearly suffices to prove the following: If

$$
\Delta=(a, b) \text { and } \Delta^{\prime}=\left(s_{0}, \ldots, s_{n}\right)
$$

where $a=s_{0}<s_{1}<\cdots<s_{n}=b$, then $Q_{\Delta} \leq \Delta_{\Delta^{\prime}}$. In this case, however,

$$
\begin{aligned}
Q_{\Delta} & =E(-\infty, a] \otimes P(a, b] \\
& =\sum_{i=1}^{n} E(-\infty, a] \otimes P\left(s_{i-1}, s_{i}\right] \\
& \leq \sum_{i=1}^{n} E\left(-\infty, s_{i-1}\right] \otimes P\left(s_{i-1}, s_{i}\right]=Q_{\Delta^{\prime}} .
\end{aligned}
$$

Proposition 6. $Q=\lim _{\Delta \in J} Q_{\Delta}$, in the strong operator topology.

Proof. Example 2 shows that every product vector in $\mathscr{H}(-\infty, a] \otimes$ $\mathfrak{S}_{\mathfrak{g}}(a, b]$ is a n.a.t. It follows that (cf. (6)) $\operatorname{ran} Q_{\Delta} \subseteq \operatorname{ran} Q$ for all $\Delta$ in $J$; i.e., $Q_{\Delta} \leq Q$ for all $\Delta$ in $J$.

Since $Q$ and each $Q_{\Delta}$ are projections, it suffices to show that $Q_{\Delta} \rightarrow Q$ weakly. Further, since $Q_{\Delta} \leq Q$ for all $\Delta$, and since the $Q_{\Delta}$ 's are uniformly bounded, it is enough to show that $\left\langle Q_{\Delta} \Psi, \phi\right\rangle \rightarrow\langle Q \Psi, \phi\rangle$ for all $\phi$ in $\Re$ and for all $\Psi$ belonging to some total set of vectors in $\mathscr{H} \otimes \mathfrak{S}$.

Observe that $\left\{f \otimes x: f \in \mathscr{H}(-T, T], x \in \mathfrak{S}_{\mathcal{B}}(-T, T], T>0\right\}$ is a total set of vectors in $\mathscr{H} \otimes \mathscr{S}$. What we shall prove is that $\left\langle Q_{\Delta}(f \otimes x), \phi\right\rangle$ $\rightarrow\langle Q(f \otimes x), \phi\rangle$ for all $\phi$ in $\mathfrak{R}$, where $f \in \mathscr{H}(-T, T]$ and $x \in \mathfrak{S}(-T, T]$ for some $T>0$.

Let $\varepsilon>0$ be given. Since $t \mapsto\left\|E_{t} f\right\|^{2}$ is monotone and uniformly continuous (recall that $\mu$ is non-atomic, and so the above function is continuous and constant in each of the intervals $(-\infty, T]$ and $(T, \infty)$ ), there exists $\Delta_{0}=\left(s_{0}, \ldots, s_{N}\right)$ in $J$ such that

(i) $s_{0}=-T ; s_{N}=T$, and 
(ii) $\left\|E_{t} f\right\|^{2}-\left\|E_{t^{\prime}} f\right\|^{2}<\varepsilon^{2} /\|x\|^{2}\|\phi\|$ whenever

$$
s_{i-1} \leq t^{\prime}<t \leq s_{i}, \quad \text { for } i=1, \ldots, N \text {. }
$$

Claim. $\left.\Delta_{0} \leq \Delta \Rightarrow K\left(Q_{\Delta}-Q\right)(f \otimes x), \phi\right\rangle \mid<\varepsilon$.

Suppose $\Delta=\left(t_{0}, \ldots, t_{n}\right) \geq \Delta_{0}$. Then $t_{0} \leq s_{0}=-T$ and $t_{n} \geq s_{N}=T$ and hence,

$$
x=P\left(t_{0}, t_{n}\right] x=\sum_{i=1}^{n} P\left(t_{i-1}, t_{i}\right] x ;
$$

thus,

$$
\begin{aligned}
\langle Q(f \otimes x), \phi\rangle & =\langle f \otimes x, \phi\rangle \quad(\text { since } \phi \in \Re) \\
& =\sum_{i=1}^{n}\left\langle f \otimes P\left(t_{i-1}, t_{i}\right] x, \phi\right\rangle \\
& =\sum_{i=1}^{n}\left\langle\left(1_{\mathscr{H}} \otimes P\left(t_{i-1}, t_{i}\right]\right)(f \otimes x), \phi\right\rangle \\
& =\sum_{i=1}^{n}\left\langle(f \otimes x),\left(1_{\mathscr{H}} \otimes P\left(t_{i-1}, t_{i}\right]\right) \phi\right\rangle \\
& =\sum_{i=1}^{n}\left\langle\left(f \otimes x, E_{t_{i}} \otimes P\left(t_{i-1}, t_{i}\right]\right) \phi\right\rangle \quad \text { (by Proposition 4) } \\
& =\sum_{i=1}^{n}\left\langle\left(E_{t_{i}} \otimes P\left(t_{i-1}, t_{i}\right]\right)(f \otimes x), \phi\right\rangle,
\end{aligned}
$$

while, by definition,

$$
\left\langle Q_{\Delta}(f \otimes x), \phi\right\rangle=\sum_{i=1}^{n}\left\langle\left(E_{t_{t-1}} \otimes P\left(t_{i-1}, t_{i}\right]\right)(f \otimes x), \phi\right\rangle .
$$

Hence

$$
\begin{aligned}
\left|\langle Q(f \otimes x), \phi\rangle-\left\langle Q_{\Delta}(f \otimes x), \phi\right\rangle\right| \\
=\left|\sum_{i=1}^{n}\left\langle\left(\left(E_{t_{i}}-E_{T_{t-1}}\right) \otimes P\left(t_{i-1}, t_{i}\right]\right),(f \otimes x), \phi\right\rangle\right| \\
=\mid \sum_{i=1}^{n}\left\langle\left(\left(E_{t_{i}}-E_{t_{t-1}}\right) \otimes P\left(t_{i-1}, t_{i}\right]\right)(f \otimes x),\right. \\
\quad\left\langle\left(1_{\mathscr{H}} \otimes P\left(t_{i-1}, t_{i}\right]\right) \phi\right\rangle \mid
\end{aligned}
$$




$$
\begin{aligned}
& \leq \sum_{i=1}^{n}\left\|\left(\left(E_{t_{i}}-E_{t_{i-1}}\right) \otimes P\left(t_{i-1}, t_{i}\right]\right)(f \otimes x)\right\| \\
& \cdot\left\|\left(1_{\mathscr{H}} \otimes P\left(t_{i-1}, t_{i}\right]\right) \phi\right\| \\
& \leq\left[\sum_{i=1}^{n}\left\|\left(E_{t_{i}}-E_{t_{i-1}}\right) f \otimes P\left(t_{i-1}, t_{i}\right] x\right\|^{2}\right]^{1 / 2} \\
& \quad \cdot\left[\sum_{i=1}^{n}\left\|\left(1_{\mathscr{H}} \otimes P\left(t_{i-1}, t_{i}\right]\right) \phi\right\|^{2}\right]^{1 / 2} \cdot \\
& \text { The first term }=\left[\sum_{i=1}^{n}\left\|\left(E_{t_{i}}-E_{t_{i-1}}\right) f\right\|^{2}\left\|P\left(t_{i-1}, t_{i}\right] x\right\|^{2}\right]^{1 / 2} \\
& \leq\left[\frac{\varepsilon^{2}}{\|x\|^{2}\|\phi\|^{2}} \sum_{i=1}^{n}\left\|P\left(t_{i-1}, t_{i}\right] x\right\|^{2}\right]^{1 / 2}=\varepsilon\|\phi\|^{-1},
\end{aligned}
$$

the first inequality being a consequence of the choice of $\Delta_{0}$, the inequality $\Delta \geq \Delta_{0}$ and the assumption $f \in \mathscr{H}(-T, T]$, while the last equality follows from $x \in \mathfrak{g}[-T, T]$.

The second term is dominated by $\|\phi\|$ since $\left\{1_{\mathscr{H}} \otimes P\left(t_{i-1}, t_{i}\right]: i=\right.$ $1, \ldots, n\}$ is a set of mutually orthogonal projections; hence, the proof of the claim, and consequently, the proof of the proposition, is complete.

The next result is an easy consequence of the last proposition.

Proposition 7. Simple n.a.t.s (cf. Definition 3) are dense in $\mathfrak{R}$.

Proof. It is to be proved that $\mathfrak{R}=\mathfrak{R}_{0}$, where $\mathfrak{M}_{0}$ is the closure of the set of simple n.a.t.s.

To start with, note that if $f \in \mathscr{H}$ and $x \in \mathfrak{B}$, then $Q_{\Delta}(f \otimes x)$ is a simple n.a.t. for every $\Delta$ in $J$, and so, by Proposition 6 , it follows that $Q(f \otimes x) \in \mathfrak{N}_{0}$.

Since $\mathscr{H} \otimes \mathscr{E}=\operatorname{sp}\{f \otimes x: f \in \mathscr{H}, x \in \mathfrak{S}\}$ it follows (from the linearity and continuity of $Q$ ) that

$$
\mathfrak{R}=Q(\mathscr{H} \otimes \mathfrak{Q})=\operatorname{sp}\left\{Q(f \otimes x): f \in \mathscr{H}, x \in \mathfrak{S}_{\mathcal{E}}\right\} \subseteq \mathfrak{N}_{0}
$$

the last inclusion following from the previous paragraph. Since, clearly, $\mathfrak{R}_{0} \subseteq \mathfrak{R}$, the proof is complete.

Observe that $\Omega \otimes x \in \mathfrak{N}$ for any $x$ in $\mathfrak{S}_{\varepsilon}$, since $E_{t} \Omega=\Omega$ for all $t$. 
THEOREM 8. There exists a unique isometic operator $\mathscr{I}: \mathfrak{R} \rightarrow \mathscr{H}$ such that

(i) $\mathscr{I}(\Omega \otimes x)=W x$ for all $x$ in $\mathfrak{S}$; and more generally,

(ii) if $a \in \mathbf{R}, f \in \mathscr{H}(-\infty, a], x \in \mathfrak{S}_{(}(a, \infty)$ and $\phi=f \otimes x$, then $\mathscr{I}_{\phi}=$ $U_{(-\infty, a],(a, \infty)}(f \otimes W x)$.

(Note: This is the analogue of the Itô integral and it is tempting to write $\mathscr{I}_{\boldsymbol{\phi}}=\int \phi d W$.)

Proof. Since elementary n.a.t.s span $\mathfrak{N}$, it is clear that (ii) forces uniqueness of $\mathscr{I}$, so it suffices to prove existence.

For typographical economy, let us write $U_{a}$ for $U_{(-\infty, a],(a, \infty)}$ and $U_{a, b}$ for $U_{(-\infty, a],(a, b]}$ when $a \leq b$, where the $U_{L, M}$ 's are as defined in (b) of Preliminaries.

If $a, f, x$ and $\phi$ are as in (ii) above, then $W x \in \mathscr{H}(a, \infty)$ (cf. (4)) and so, it makes sense to define $\mathscr{I}_{\phi}=U_{a}(f \otimes W x)$. That $\mathscr{I}_{\phi}$ is unambiguously defined (in the sense that $\mathscr{I}_{\phi}$ depends only on $\phi$, and not on $a, f$ or $x$ ) is a consequence of the consistency properties of the $U_{L, M}$ 's stated in Proposition (U).

Next suppose $a, b \in \mathbf{R}, f \in \mathscr{H}(-\infty, a], \quad x \in \mathfrak{S}(a, \infty), \phi=f \otimes x$, and $g \in \mathscr{H}(-\infty, b], y \in \mathfrak{S}(b, \infty), \Psi=g \otimes y$. Assume (without loss of generality) that $a \leq b$. Then, observe that

$$
\begin{aligned}
\mathscr{I}_{\phi} & =\mathscr{I}(f \otimes x)=U_{a}(f \otimes W x) \\
& =U_{a}(f \otimes W(P(a, b] x+P(b, \infty) x)) \\
& =U_{a}(f \otimes W P(a, b] x)+U_{a}(f \otimes W P(b, \infty) x) .
\end{aligned}
$$

Notice that $U_{a}(f \otimes W P(a, b] x) \in \mathscr{H}(-\infty, b]$ and so,

$$
U_{a}(f \otimes W P(a, b] x)=U_{b}\left(U_{a, b}(f \otimes W P(a, b] x) \otimes \Omega\right) .
$$

Similarly

$$
U_{a}(f \otimes W P(b, \infty) x)=U_{b}\left(U_{a, b}(f \otimes \Omega) \otimes W P(b, \infty) x\right) .
$$

On the other hand, by definition,

$$
\mathscr{I} \Psi=\mathscr{I}(g \otimes y)=U_{b}(g \otimes W y) .
$$

Since $U_{b}$ is unitary, conclude that

$$
\begin{aligned}
\left\langle\mathscr{I}_{\phi}, \mathscr{I} \Psi\right\rangle= & \left\langle U_{a, b}(f \otimes W P(a, b] x) \otimes \Omega, g \otimes W y\right\rangle \\
& +\left\langle U_{a, b}(f \otimes \Omega) \otimes W P(b, \infty) x, g \otimes W y\right\rangle .
\end{aligned}
$$


The first term on the right is zero since $\langle\Omega, W y\rangle=0$ (cf. (4)), and so, since $W$ is isometric,

$$
\begin{aligned}
\left\langle\mathscr{I}_{\phi}, \mathscr{I} \Psi\right\rangle & =\left\langle U_{a, b}(f \otimes \Omega), g\right\rangle\langle P(b, \infty) x, y\rangle \\
& =\langle f, g\rangle\langle x, P(b, \infty) y\rangle \quad\left(\text { since } U_{a, b}(f \otimes \Omega)=f\right) \\
& =\langle f, g\rangle\langle x, y\rangle \quad(\text { since } y \in \mathscr{H}(b, \infty)) \\
& =\langle f \otimes x, g \otimes y\rangle=\langle\phi, \Psi\rangle .
\end{aligned}
$$

So, the equation (ii) (in the statement of the theorem) unambiguously defines a vector $\mathscr{I}_{\phi}$ in $\mathscr{H}$ for every elementary n.a.t. $\phi$; further, if $\phi$ and $\Psi$ are elementary n.a.t.s, then $\left\langle\mathscr{I}_{\phi}, \mathscr{I} \Psi\right\rangle=\langle\phi, \Psi\rangle$. Since elementary n.a.t.s generate $\mathfrak{R}$ (by Proposition 7), it is clear that $\mathscr{I}$ extends to a unique isometric operator from $\mathfrak{N}$ into $\mathscr{H}$.

Finally, we identify the range of $\mathscr{I}$, and this result is essentially the Kunita-Watanabe Theorem.

THEOREM 9. $\mathscr{I}(\mathfrak{R})=\{\Omega\}^{\perp}=\mathscr{H} \ominus \mathbf{C} \Omega$.

Proof. Since $\{\Omega\}^{\perp}=\operatorname{sp}\{\Gamma(x)-\Omega: x \in \mathscr{S}\}$, and since $\mathscr{I}$ (being isometric) has closed range, it suffices to prove the following:

Claim. $\Gamma(x)-\Omega=\mathscr{I}(Q(\Gamma(x) \otimes x))$ for all $x$ in $\mathfrak{H}$. Since $\mathscr{H}=$ $\operatorname{sp}\left\{\Gamma(y): y \in \mathfrak{S}_{\mathcal{E}}\right\}$, it is enough to establish that

$$
\langle\mathscr{I}(Q(\Gamma(x) \otimes x)), \Gamma(y)\rangle=\langle\Gamma(x)-\Omega, \Gamma(y)\rangle=\exp \langle x, y\rangle-1 .
$$

In view of Proposition 6 (and the continuity of $\mathscr{I}$ ), it is enough to prove that

$$
\lim _{\Delta \in J}\left\langle\mathscr{I}\left(Q_{\Delta}(\Gamma(x) \otimes x)\right), \Gamma(y)\right\rangle=\exp \langle x, y\rangle-1 \text {. }
$$

Let $\Delta=\left(t_{0}, \ldots, t_{n}\right) \in J$. Writing $U_{t}$ for $U_{(-\infty, t],(t, \infty)}$ (as in the proof of Theorem 8), we see that

$$
\begin{aligned}
\mathscr{I}\left(Q_{\Delta}(\Gamma(x) \otimes x)\right) & =\mathscr{I}\left(\sum_{i=1}^{n} E_{t_{i-1}}(\Gamma(x)) \otimes P\left(t_{i-1}, t_{i}\right] x\right) \\
& =\mathscr{I}\left(\sum_{i=1}^{n} \Gamma\left(P_{t_{t-1}}\right) \Gamma(x) \otimes P\left(t_{i-1}, t_{i}\right] x\right) \\
& =\mathscr{I}\left(\sum_{i=1}^{n} \Gamma\left(P_{t_{i-1}} x\right) \otimes P\left(t_{i-1}, t_{i}\right] x\right) \\
& =\sum_{i=1}^{n} U_{t_{t-1}}\left(\Gamma\left(P_{t_{i-1}} x\right) \otimes W P\left(t_{i-1}, t_{i}\right] x\right) .
\end{aligned}
$$


On the other hand, for any $t$ in $\mathbf{R}$,

$$
\Gamma(y)=U_{t}\left(\Gamma\left(P_{t} y\right) \otimes \Gamma(P(t, \infty) y)\right) .
$$

Hence,

$$
\begin{aligned}
&\left\langle\mathscr{I}\left(Q_{\Delta}(\Gamma(x) \otimes x)\right), \Gamma(y)\right\rangle= \sum_{i=1}^{n}\left\langle\Gamma\left(P_{t_{i-1}} x\right), \Gamma\left(P_{t_{i-1}} y\right)\right\rangle \\
& \cdot\left\langle W P\left(t_{i-1}, t_{i}\right] x, \Gamma\left(P\left(t_{i-1}, \infty\right) y\right)\right\rangle \\
&=\sum_{i=1}^{n}\left(\exp \left\langle P_{t_{i-1}} x, P_{t_{i-1}} y\right\rangle\right)\left\langle P\left(t_{i-1}, t_{t}\right] x, P\left(t_{i-1}, \infty\right) y\right\rangle \\
&=\sum_{i=1}^{n}\left(\exp \left\langle P_{t_{i-1}} x, y\right\rangle\right)\left\langle P\left(t_{i-1}, t_{i}\right] x, y\right\rangle \\
&=\sum_{i=1}^{n}\left(\exp \alpha\left(t_{i-1}\right)\right) \cdot\left(\alpha\left(t_{i}\right)-\alpha\left(t_{i-1}\right)\right),
\end{aligned}
$$

where $\alpha(t)=\left\langle P_{t} x, y\right\rangle$.

Hence $\left\langle\mathscr{I}\left(Q_{\Delta}(\Gamma(x) \otimes x)\right), \Gamma(y)\right\rangle$ is a typical Riemann sum (considering the left end point) corresponding to the partition $\Delta$, in the evaluation of the Riemann-Stieltje's integral $\int_{-\infty}^{\infty}(\exp \alpha(t)) d \alpha(t)$. (Note that $\alpha(t)$ is a function of finite total variation.) Taking limits as the partition is indefinitely refined, we get, by Proposition 6,

$$
\begin{aligned}
\langle\mathscr{I}(Q(\Gamma(x) \otimes x)), \Gamma(y)\rangle & =\int_{-\infty}^{\infty} e^{\alpha(t)} d \alpha(t) \\
& =\left.e^{\alpha(t)}\right|_{-\infty} ^{\infty}=e^{\langle x, y\rangle}-1, \text { as desired. }
\end{aligned}
$$

The Kunita-Watanabe theorem (cf. [4]) is stated in terms of martingales. To make contact with that formulation, one can define a martingale (in this setting) as a curve $\{\phi(t): t \in \mathbf{R}\}$ in $\mathscr{H}$ such that $E_{s} \phi(t)=\phi(s)$ for $s \leq t$. It can easily be verified, that $\phi(t)=E_{t} \mathscr{I}(\phi)$ defines a martingale with 'mean zero' for any $\phi$ in $\mathfrak{N}$ (i.e., $\left\langle E_{t} \mathscr{I}(\phi), \Omega\right\rangle=$ $0)$. It can now be deduced from Theorem 9 that if $\{\phi(t): t \in \mathbf{R}\}$ is a martingale such that (i) $\langle\phi(t), \Omega\rangle 0$ for all $t$, and (ii) $\sup _{t}\|\phi(t)\|<\infty$, then there exists $\phi$ in $\mathfrak{R}$ such that $\phi(t)=E_{t} \mathscr{I}(\phi)=\mathscr{I}\left(\left(1_{\mathscr{H}} \otimes P_{t}\right) \phi\right)$. The verification of the above details is fairly painless and we shall be content to stop here.

Acknowledgment. I wish to thank Professor K. R. Parthasarathy for introducing me to continuous tensor products, Fock space and stochastic integration. 


\section{REFERENCES}

[1] H. Araki and E. J. Woods, Complete Boolean algebras of Type-I factors, Publications of the RIMS, Kyoto University, Ser. A, 2, No. 2, (1966), 157-242.

[2] J. Dixmier, Les algebres des operateurs dans l'espace Hilbertien, Gauthier-Villars, Paris, 1957.

[3] K. Ito, Stochastic integral, Proc. Imp. Acad. Tokyo, 20 (1944), 519-524.

[4] H. Kunita and S. Watanabe, On square -integrable martingales, Nagoya Math. J., 30 (1967), 209-245.

[5] H. P. McKean, Jr., Stochastic Integrals, Academic Press, New York, 1969.

[6] K. R. Parthasarathy and K. Schmidt, Positive Definite Kernels, Continuous Tensor Products and Central Limit Theorems in Probability Theory, Lecture Notes in Mathematics, No. 272, Springer-Verlag, Berlin, 1970.

Received September 20, 1984.

INDIAN STatistical INSTITUTE

DELHI CENTRE

7, S.J.S. Sansanwal Marg.

NEW DELHI - 110016 INDIA 



\section{PACIFIC JOURNAL OF MATHEMATICS EDITORS}

\author{
V. S. VARAdarajan (Managing Editor) \\ University of California \\ Los Angeles, CA 90024 \\ Hebert Clemens \\ University of Utah \\ Salt Lake City, UT 84112 \\ Charles R. DePrima \\ California Institute of Technology \\ Pasadena, CA 91125
}

R. FINN

Stanford University

Stanford, CA 94305

HeRManN FLASChKa

University of Arizona

Tucson, AZ 85721

RAMESH A. GANGOlli

University of Washington

Seattle, WA 98195

ROBION KIRBY

University of California

Berkeley, CA 94720

\author{
C. C. MOORE \\ University of California \\ Berkeley, CA 94720 \\ H. SAMELSON \\ Stanford University \\ Stanford, CA 94305 \\ HAROLD STARK \\ University of California, San Diego \\ La Jolla, CA 92093
}

\section{ASSOCIATE EDITORS}

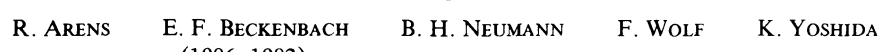

(1906-1982)

\section{SUPPORTING INSTITUTIONS}

\begin{abstract}
UNIVERSITY OF ARIZONA
UNIVERSITY OF BRITISH COLUMBIA

CALIFORNIA INSTITUTE OF TECHNOLOGY

UNIVERSITY OF CALIFORNIA

MONTANA STATE UNIVERSITY

UNIVERSITY OF NEVADA, RENO

NEW MEXICO STATE UNIVERSITY

OREGON STATE UNIVERSITY
\end{abstract}

\author{
UNIVERSITY OF OREGON \\ UNIVERSITY OF SOUTHERN CALIFORNIA \\ STANFORD UNIVERSITY \\ UNIVERSITY OF HAWAII \\ UNIVERSITY OF TOKYO \\ UNIVERSITY OF UTAH \\ WASHINGTON STATE UNIVERSITY \\ UNIVERSITY OF WASHINGTON
}

The Supporting Institutions listed above contribute to the cost of publication of this Journal, but they are not owners or publishers and have no responsibility for its content or policies.

Mathematical papers intended for publication in the Pacific Journal of Mathematics should be in typed form or offset-reproduced (not dittoed), double spaced with large margins. Please do not use built up fractions in the text of the manuscript. However, you may use them in the displayed equations. Underline Greek letters in red, German in green, and script in blue. The first paragraph must be capable of being used separately as a synopsis of the entire paper. In particular it should contain no bibliographic references. Please propose a heading for the odd numbered pages of less than 35 characters. Manuscripts, in triplicate, may be sent to any one of the editors. Please classify according to the scheme of Math. Reviews, Index to Vol. 39. Supply name and address of author to whom proofs should be sent. All other communications should be addressed to the managing editor, or Elaine Barth, University of California, Los Angeles, California 90024.

There are page-charges associated with articles appearing in the Pacific Journal of Mathematics. These charges are expected to be paid by the author's University, Government Agency or Company. If the author or authors do not have access to such Institutional support these charges are waived. Single authors will receive 50 free reprints; joint authors will receive a total of 100 free reprints. Additional copies may be obtained at cost in multiples of 50 .

The Pacific Journal of Mathematics is issued monthly as of January 1966. Regular subscription rate: $\$ 190.00$ a year (5 Vols., 10 issues). Special rate: $\$ 95.00$ a year to individual members of supporting institutions.

Subscriptions, orders for numbers issued in the last three calendar years, and changes of address should be sent to Pacific Journal of Mathematics, P.O. Box 969, Carmel Valley, CA 93924, U.S.A. Old back numbers obtainable from Kraus Periodicals Co., Route 100, Millwood, NY 10546.

The Pacific Journal of Mathematics at P.O. Box 969, Carmel Valley, CA 93924 (ISSN 0030-8730) publishes 5 volumes per year. Application to mail at Second-class postage rates is pending at Carmel Valley, California, and additional mailing offices. Postmaster: Send address changes to Pacific Journal of Mathematics, P.O. Box 969, Carmel Valley, CA 93924.

PUBLISHED BY PACIFIC JOURNAL OF MATHEMATICS, A NON-PROFIT CORPORATION

Copyright $\odot 1986$ by Pacific Journal of Mathematics 


\section{Pacific Journal of Mathematics}

\section{Vol. 122, No. 2 \\ February, 1986}

Gideon Amit and David Chillag, On a question of Feit concerning character values of finite solvable groups ......................257

Constantin Gelu Apostol and Frank Larkin Gilfeather, Isomorphisms modulo the compact operators of nest algebras ................263

Parviz Azimi and James Neil Hagler, Examples of hereditarily $l^{1}$ Banach

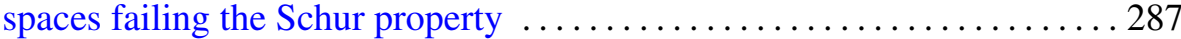

Brian Evan Blank, Boundary behavior of limits of discrete series representations of real rank one semisimple groups . . . . . . . . . . 299

Jeffrey Carroll, Some undecidability results for lattices in recursion theory

Gerald Howard Cliff and Alfred Rheinhold Weiss, Crossed product and

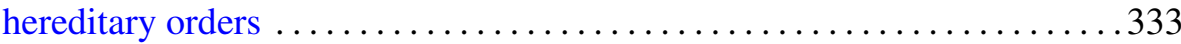

Ralph Cohen, Realizing transfer maps for ramified coverings . . . . . . . . 347

Ronald James Evans, Hermite character sums . .................. 357

C. L. Frenzen and Roderick Sue-Chuen Wong, Asymptotic expansions of the Lebesgue constants for Jacobi series . . . . . . . . . . . . . . . . 391

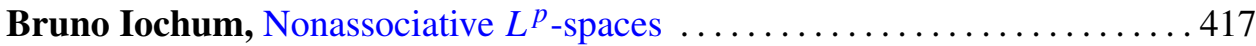

John McDonald, Unimodular approximation in function algebras ....... 435

John Robert Quine, Jr., Ramification and unintegrated value distribution . . 441

Marc Raphael, Commutants of quasisimilar subnormal operators ........ 449

Parameswaran Sankaran and Peter Zvengrowski, On stable

parallelizability of flag manifolds

Helga Schirmer, A relative Nielsen number

Barry Simon, Schrödinger semigroups on the scale of Sobolev spaces . . . . . 475

Viakalathur Shankar Sunder, Stochastic integration in Fock space

Jan de Vries, A note on the $G$-space version of Glicksberg's theorem 